\title{
ESTUDO BRASILEIRO DE NUTRIÇÃO E SAUDE (EBANS): OPORTUNIDADES E DESAFIOS METODOLOGICOS
}

\section{Pôster}

Autores deste trabalho:

Ana Del Arco: Departamento de Pediatria, Escola Paulista de Medicina, Universidade Federal de São Paulo, São Paulo, Brasil

Mauro Fisberg: - Departamento de Pediatria, Escola Paulista de Medicina, Universidade Federal de São Paulo, São Paulo, Brasil - Instituto Pensi, Fundação Jose Luiz Egydio Setubal, Sabará Hospital Infantil, São Paulo, Brasil

Ágatha Nogueira Previdelli: Faculdade de Ciências Biológicas e da Saúde, Universidade São Judas Tadeu, São Paulo, Brasil

Aline Veroneze de Mello: Departamento de Nutrição, Faculdade de Saúde Pública, Universidade de São Paulo, São Paulo, Brasil

Irina Kovalskys: Committee of Nutrition and Wellbeing, International Life Science Institute (ILSI-Argentina), Buenos Aires, Argentina

Área do Trabalho: Nutrição

Data da submissão: 30/07/2018 às 12:11

\section{Justificativa}

A obesidade atinge quase $1 / 3$ da população mundial e agrega consequências para a saúde que são observadas na infância e adolescência. De ordem multifatorial, ela deve ser enfrentada sob diversos aspectos, no qual a alimentação e a atividade física destacam-se por serem fatores associados modificáveis. Objetivando evitar o agravamento de uma determinada condição de saúde, estudos epidemiológicos, como se propõe o Estudo Brasileiro de Nutrição e Saúde (EBANS), são fundamentais para diagnosticar o estado nutricional da população.

\section{Objetivo(s)}

Evitar o agravamento de uma determinada condição de saúde (através da identificação de variáveis relacionadas ao desfecho); realizar um diagnóstico do estado nutricional da população brasileira (nas cinco regiões do país, com indivíduos de 15 a 65 anos), bem como dos parâmetros associados à obesidade, com diversas possibilidades de correlacionar dados.

\section{Método(s)}

Parte do estudo ELANS (Estudo Latino-Americano de Nutrição e Saúde) (n=9218), o EBANS ( $n=2000)$ é um estudo de base populacional cujos dados permitem: avaliar o nível socioeconômico; realizar diagnóstico do estado nutricional (peso corporal, estatura, índice de massa corporal, circunferências de pescoço, cintura e quadril); avaliar consumo alimentar (R24h e QFA para bebidas) e avaliar prática de atividade física (IPAQ-longo e acelerômetro). De abrangência nacional, o EBANS tem potencial para compor análises regionais, além de retratar a atual condição epidemiológica nutricional, de consumo alimentar e padrão de atividade física da população brasileira, em diferentes estágios da vida, podendo ter seus dados analisados em 
conjunto ou estratificados (por faixas etárias, nível socioeconômico e gênero), oferecendo subsídios úteis para a formulação de políticas públicas. Registro CAAE: 31670314.8.0000.5567.

\section{Resultado(s)}

Cada etapa metodológica foi desenhada a fim de reduzir os erros e vieses atrelados aos desafios metodológicos.

\section{Conclusão (ões)}

Com grande potencial para futuras análises de dados, o EBANS intenta contribuir na geração de conhecimento para fomentar políticas e ações capazes de alterar o atual cenário de obesidade. 